\title{
Análise e melhoria de processos operacionais em cooperativas: Uma abordagem estatística
}

\author{
Analysis and improvement of operational processes in cooperatives: \\ a statistical approach
}

\begin{abstract}
Resumo:
O negócio cooperativo tem ganhado espaço na economia atual com vistas à proporcionar alianças entre os cooperados e maior impacto social junto à sociedade. Muitos agricultores têm se unido em cooperativas com o intuito de valorizar ainda mais seus produtos frente ao mercado e a formação de indústrias de beneficiamento tem se mostrado bastante frutífera. Estas indústrias transformam a matéria-prima comum dos produtores em uma gama maior de produtos, agregando valor e obtendo maior lucratividade, além de desenvolver a região em que está inserida. Neste sentido, o objetivo deste trabalho é analisar estatisticamente resultados obtidos nos processos operacionais que visam a distribuição e devolução dos produtos de uma cooperativa do Rio Grande do Sul, com vistas á geração de subsídios para a melhoria destes processos. Dentre os problemas mais críticos encontrados estão deficiências nos processos de transporte e armazenamento e na emissão de notas fiscais, gerando grande número de devoluções.
\end{abstract}

Palavras-chave: Cooperativas, processos operacionais, estatística descritiva.

\begin{abstract}
:
The cooperative business has gained ground in the current economy with a view to providing alliances between cooperative and greater social impact in society. Many farmers have joined in cooperatives in order to further enhance its products outside the market and the formation of processing industries has been very fruitful. These industries transform common raw material producers in a wider range of products, adding value and achieving greater profitability, and develop the region in which it operates. In this sense, the objective is statistically analyze results obtained in the management processes of a cooperative of Rio Grande do Sul, with a view will generate subsidies for the improvement of these processes. Among the most critical problems are found deficiencies in transport processes and storage and issuing invoices, generating large number of returns.
\end{abstract}

Keywords: Cooperatives, operational processes, descriptive statistics.

Recebido: 06/07/2015 Aceito: 06/01/2016

Deise Graziele Dickel ${ }^{1}$ e Julio Cezar Mairesse Siluk ${ }^{2}$

${ }^{1}$ Universidade Federal de Santa Maria , Mestrado em Engenharia de Produção - deisedickelsm@ gmail.com -

Rua Cristalino Machado Soares, no 211 fundos, Bairro Camobi, Santa Maria - RS. CEP 97110210

${ }^{2}$ Universidade Federal de Santa Maria, Doutorado em Engenharia de Produção - jsiluk@ufsm.com.br 


\section{Introdução}

$\mathrm{O}$ movimento cooperativo surgiu no século XIX, em resposta a grande desvantagem social de algumas camadas da sociedade e no interesse dos trabalhadores, em sua maioria pobres (MORI, 2014). Este movimento compreendeu que a união de pessoal em prol de objetivos comuns, sejam eles pessoais ou econômicos, também pode ser uma maneira de ampliar a lucratividade das atividades desenvolvidas e deu-se as uniões de pessoas com fins comuns o nome de cooperativas (BRUSSELAERS, POPPE e AZCARATE, 2014). Essencialmente, define-se a cooperativa como uma associação de pessoas, em bases democráticas, que se unem com o objetivo de atender a certas necessidades econômicas fundamentais, manifestando duas dimensões básicas: de instituição política, interessada na organização e promoção social de seus membros e, ao mesmo tempo, um empreendimento econômico que se obriga a produzir algum bem ou serviço dentro de um grau relativo de eficiência econômica (ANTONIALLI, 2000).

Em todo o mundo, as cooperativas têm sido importantes instituições para reforçar a posição econômica dos agricultores, uma vez que esta categoria precisa estar envolvida na constante negociação com os vendedores de insumos agrícolas ou compradores de produtos agrícolas. Nas últimas décadas, a concentração entre os varejistas de alimentos tem reforçado a necessidade de construir uma estratégia que beneficie os pequenos agricultores. Outra importante função das cooperativas refere-se à redução dos custos de transação na relação de vendas entre os agricultores e os seus clientes. Com a crescente importância do controle de qualidade e garantia de qualidade tornaram-se cada vez mais necessário, devido a mais rigorosos requisitos públicos e privados (BIJMAN e ILIOPOULOS, 2014).

As cooperativas também desempenham o papel de coordenador na garantia de alta qualidade na cadeia de abastecimento, uma vez que centralizam os produtos e são responsáveis por coloca-los no mercado (BIJMAN e ILIOPOULOS, 2014). Além disto, há muitos outros aspectos associados ao desempenho da cooperativa, tais como a satisfação dos membros, preços pagos pelos produtos dos membros, quota de mercado e satisfação dos clientes (SOBOH et al., 2009).
Dentro deste contexto, busca-se alternativas para aumentar os lucros e permitir o crescimento das cooperativas. O controle e a redução de erros da produção e de logística tem se mostrado uma boa opção para o atingimento deste objetivo que é comum às cooperativas e as demais empresas. Para se obter um parâmetro dos erros cometido é necessário um registro preciso de ocorrências de problemas, bem como suas causas. A partir dessas informações é possível efetuar estudos para melhorar tal situação.

Neste sentido, o presente trabalho vem colaborar com o estudo dos processos operacionais que visam a distribuição e devolução dos produtos de uma cooperativa do Rio Grande do Sul, com o objetivo de analisar e melhorar estes processos por meio da aplicação da estatística descritiva.

A Cooperativa estudada, será chamada pelo nome fictício de "Cooperativa Beta" e tem associadas as principais cooperativas agropecuárias gaúchas, o que representa um universo de 171.000 produtores rurais, em mais de 350 municípios do Rio Grande do Sul, atuando no beneficiamento de leite para produção de leite em pó e derivados, bem como a centralização logística para exportação de grãos.

\section{Gestão por processos}

Muitas empresas já conhecem e se valem da gestão por processos para melhorar os seus resultados operacionais. Davenport (1993) afirma que processo é uma organização de atividade de trabalho, com início, fim e com entradas e saídas claramente definidas. Para Beretta (2002), processos indicam onde os recursos e competências da empresa são ativados a fim de criar uma competência organizacional capaz de preencher suas lacunas para gerar uma vantagem competitiva sustentável (PÁDUA, 2012).

As cooperativas, por sua vez também, estão descobrindo os benefícios proporcionados pela Business Process Management (BPM), que busca o alcance dos objetivos organizacionais através da melhoria, gestão e controle de processos essenciais (JESTON; NELIS, 2006; IRITANI et al, 2015). De acordo com Palmberg (2010), os motivos que levam uma organização a buscar o BPM são particulares a cada organização. Entretanto, é possível identificar os motivos mais comuns (MÜCKENBERGER, 2013): 
- A organização apresenta crescimento acentuado levando a dificuldades em atingir seus objetivos e perda de controle e agilidade para aproveitar oportunidades;

- Há dificuldades no gerenciamento, com informações imprecisas e/ou conflitantes e necessidade de cortar custos e aumentar produtividade;

- Ocorre alta rotatividade, falta de preparo e insatisfação dos colaboradores;

- Há aumento no número de clientes, fornecedores ou parceiros e elevam-se as exigências em atendêlos através de um relacionamento de proximidade;

- Os produtos e serviços oferecidos são complexos e há duplicação de tarefas;

- Não há visão dos processos ponta a ponta, gerando lacunas e falta de padronização e clareza quanto a responsabilidades; e

- Ocorrem introdução ou eliminação de sistemas de informação.

Para melhor identificar e estabelecer prioridades de trabalho entre processos, Oliveira (2009) sugere que os processos primários sejam classificados entre processos-chave e processos críticos. Os processos-chave são os que têm custo elevado para a organização e alto impacto para o cliente. Já os processos críticos são os processos-chave que se relacionam diretamente com a estratégia organizacional.

Para organizar a empresa por processos de negócio, precisamos colocar o foco no cliente externo, já que os processos de começam e terminam nele. Os processos enxergam uma linha de atividades que começa com o entendimento exato do que o cliente externo deseja e termina com o cliente externo adquirindo o que ele precisa e deseja de um negócio (GONÇALVES, 2000).

A noção de valor para o cliente é baseada na percepção da vantagem ou do benefício que ele recebe em cada transação com a empresa. Essa percepção depende, por exemplo, da relação entre o tempo de processamento e o tempo de ciclo. O preço pago é apenas uma parte do esforço para obter o produto ou serviço. $\mathrm{O}$ cliente avalia também a rapidez e o conforto para obter o produto e a oportunidade de conseguir o que deseja. Nem sempre o valor para o cliente é identificado de maneira clara e indiscutível, e sua avaliação é difícil, já que existem componentes irracionais e emocionais (GONÇALVES, 2000).

\section{Análise com base na estatística descritiva}

A estatística descritiva tem por objetivo descrever os dados observados (SILVA, 1999) e ainda organizar, compreender e facilitar o entendimento do significado das variáveis através da redução dos números brutos. São considerados dados brutos a sequência de todas as $n$ observações diretas de um fenômeno qualquer. A ordenação desses dados em uma sequência é denominado Rol.

Através da utilização de planilhas eletrônicas ou pacotes computacionais é possível obter um conjunto de medidas descritivas provenientes dos valores de variáveis quantitativas (BARBETTA, 2004). As medidas de tendência central são valores que representam uma entrada central do conjunto de dados, as mais usuais são a média, a mediana e a moda (LARSON, 2010). A média de um conjunto de dados amostrais é a soma dos valores observados pela razão do número de observações conforme Fórmula (1):

$$
\bar{x}=\frac{\sum x i}{n}
$$

A mediana representa o valor que está no meio do rol de dados, ou seja, mede o centro do conjunto dividindo-o em duas partes iguais. Caso o conjunto tenha um número ímpar de dados a mediana será o termo do meio (2) e se for um número par, a mediana será a média das entradas do meio (3).

$$
\begin{aligned}
& M d=\frac{n+1}{2} \\
& M d=\frac{n}{2} \text { e } M d=\frac{n}{2}+1
\end{aligned}
$$

A moda de um conjunto representa o valor de entrada que ocorre com maior frequência. Nos casos em que há duas entradas com frequências idênticas diz-se que o conjunto é bimodal, da mesma forma que se os dados não se repetirem, dizse que este é amodal.

As medidas de dispersão tem a função de descrever os dados e informar o grau de dispersão dos valores observados em relação a uma medida de tendência central, ou seja, o afastamento. Essas medidas indicam a homogeneidade do conjunto, quando possuem pouca ou variabilidade nula ou a heterogeneidade, muita variabilidade dos dados. Algumas medidas de dispersão são: variância, desvio padrão, coeficiente de variação e amplitude 


\section{(LARSON, 2010).}

A variância consiste na média aritmética dos desvios quadrados (4), através do cálculo da variância analisa-se o grau de variabilidade dos dados. Como sua unidade de medida é quadrada, a extração da raíz resulta no desvio padrão (5). O uso da desvio padrão é vantajoso em relação a variância pois este permite que as análises sejam feitas de forma direta, já que, a unidade de medida é a mesma dos dados observados.

$$
\begin{aligned}
& s^{2}=\frac{1}{n-1} \sum_{i=1}^{n}(x i-\bar{x})^{2} \\
& s=\sqrt{\frac{1}{n-1} \sum_{i=1}^{n}(x i-\bar{x})^{2}}
\end{aligned}
$$

O erro padrão é calculado (6) pela razão do desvio padrão pela raíz quadrada $\mathrm{n}$, que significa o número de observações. Esse erro estima a variabilidade das médias.

$s=\frac{s}{\sqrt{n}}$

O intervalo dado é o que se pode definir como amplitude (7), quer dizer, é a diferença entre o maior e o menor elemento de um sequência.

$$
H=X m a ́ x-X m i n
$$

Coeficiente de assimetria indica a posição da cauda no gráfico de distribuição, ou seja, o grau de deformação da distribuição (8). Quando o coeficiente é positivo indica que a cauda é para o lado direito, quando é negativo indica que a cauda é para o lado esquerdo e quanto mais próximo de zero indica que a distribuição é simétrica (BARBETTA, 2004). A assimetria define-se pelo grau de desvio de uma distribuição de frequência.

$C . A .=\frac{\bar{x}-M o}{s}$

A curtose é o grau de afilamento de uma curva em relação da curva considerada normal. Uma distribuição pode ser classificada em platicúrtica, que é mais achatada do que a normal (possui desvios grandes), mesocúrtica é a curva normal (os desvios são intermediários) e leptocúrtica em que a curva é a mais alta do que a normal (os desvios são pequenos). Para medir a curtose é feito o coeficiente centílico de curtose (9).

$$
K=\frac{Q_{3}-Q_{1}}{2\left(D_{9}-D_{1}\right)}
$$

Em que $\mathrm{Q}_{1}$ é o primeiro quartil, $\mathrm{Q}_{3}$ é o terceiro quartil, $\mathrm{D}_{1}$ é o primeiro decil e o $\mathrm{D}_{9}$ é o nono decil.

A estatística descritiva auxilia a descrever os dados de uma forma mais sucinta com o auxílio das medidas de tendência central ou de dispersão, além de interpretar e apresentar as informações que se fizerem necessárias (BARBETTA, 2004; LARSON, 2010).

\section{Metodologia}

Quanto à natureza, essa pode ser considerada como aplicada devido a resposta prática obtida por seus resultados para as organizações, contribuindo para a solução de problemas pontuais no contexto e no momento da investigação (GIL, 2010).

Em relação à abordagem, essa contemplará tanto o viés qualitativo quanto o quantitativo, pois se buscará por informações que permitam evidenciar estatisticamente as características das dificuldades encontradas na indústria e posteriormente, realizar uma análise qualitativa destes dados para auxílio no processo de melhoria (MARCONI; LAKATOS, 2010).

Quanto aos procedimentos técnicos adotados, a pesquisa utilizará três desses para o pleno cumprimento dos seus objetivos: por um lado, fará uso da pesquisa bibliográfica, devido à necessidade de se buscar por publicações científicas que embasam as temáticas abordadas; por outro, utilizará a pesquisa documental para possibilitar um melhor entendimento da organização; e por último, enquadra-se como um estudo de caso, por se tratar do estudo de uma única cooperativa (GIL, 2010; YIN, 2010).

Para o desenvolvimento da pesquisa, foram realizadas entrevistas com gestores e também foram coletados os dados de fonte documental referentes as ocorrências de devolução de produtos por parte dos clientes da cooperativa. Foram monitoradas 761 devoluções no período de 14 de dezembro de 2011 a 17 de julho de 2013. Aos dados foram aplicadas as análises estatísticas descritivas, que, apesar de fácil aplicação, podem oferecer uma gama extensa de informações a serem trabalhadas.

A análise dos dados foi realizada com auxílio de planilha eletrônica e os resultados obtidos foram remetidos à cooperativa para que a mesma pudesse 
tomar ações que melhorassem seu desempenho nos aspectos mais críticos.

\section{Resultados e discussões}

A Cooperativa Beta, provem da união de produtores rurais com o objetivo de alavancar o agronegócio e principalmente gerar diferencial competitivo aos produtos. Esse diferencial foi proposto através do beneficiamento da matéria-prima que anteriormente era repassada para a indústria em sua forma natural.

$\mathrm{Na}$ unidade de lácteos da cooperativa é efetuada a produção dos derivados do leite, por meio do uso de modernas instalações e tecnologias de fabricação, em que o foco é o desenvolvimento de produtos de alta qualidade e com origem conhecida. Por isso, com o auxílio da unidade de logística, o transporte da matéria-prima fica a cargo da própria Cooperativa. Esta operação garante a qualidade dos produtos e sua confiabilidade no mercado, além disso, conforto e praticidade aos produtores associados, já que o transporte da produção é feito de maneira padronizada e segura.

Atualmente, a Cooperativa Beta tem associadas as principais cooperativas agropecuárias gaúchas, $o$ que representa um universo de 171.000 produtores rurais, em mais de 350 municípios do Rio Grande do Sul.

A cooperativa preocupa-se em atender bem seus clientes, por isso investe na inovação de produtos e embalagens. Também conta com a associação a outras cooperativas agropecuárias para oferecer aos produtores associados insumos de qualidade e com preços competitivos.

Contudo, entrevistas com gestores da Cooperativa Beta mostraram a ocorrência de alguns problemas que causam perdas e acabam reduzindo os ganhos finais da empresa. O grande número de devoluções de produto por parte dos clientes foi apontado como um dos principais problemas, pois a devolução gerada provoca perda do produto, custos com logística, descarte e também a insatisfação dos clientes. Principalmente a entrega incorreta ou fora do período contratado descontentam os clientes e podem inclusive abalar a imagem da indústria deixando a concorrência em vantagem que pode ser muito difícil de reverter.

Por este motivo, a cooperativa Beta buscou registrar todos os pedidos de devolução de produtos com vistas à compreender melhor o tamanho da perda e os motivos que causam estas devoluções. Durante o período de 14 de dezembro de 2011 a 17 de julho de 2013 foram efetuados apontamentos das devoluções de pedidos por parte dos clientes com o objetivo de análise e redução desses acontecimentos. Esse registro discrimina detalhadamente itens como, o tipo de produto devolvido, a quantidade e a razão da devolução em um relatório em planilha eletrônica. Foram registradas no período 760 devoluções de 19 produtos distintos, considerando que um mesmo produto pode ter várias embalagens.

Tabela 1: Motivos de ocorrências de devolução

\begin{tabular}{lcc}
\hline Ocorrência & Total & Proporção \\
\hline 1 - Recusa por atraso na entrega & 3 & 0,0039 \\
2 - Avaria no transporte & 226 & 0,2974 \\
3 - Sinistro & 3 & 0,0039 \\
4 - Emissão nota fiscal incorreta & 180 & 0,2368 \\
5 - Desistência da compra pelo cliente & 18 & 0,0237 \\
6 - Devolução de produto vencido & 8 & 0,0105 \\
7 - Devolução de produto não justificado & 126 & 0,1658 \\
8 - Devolução produto por desvio qualidade & 133 & 0,1750 \\
9 - Avaria no carregamento & 49 & 0,0645 \\
10 - Troca de produto & 14 & 0,0184 \\
\hline Total Geral & 760 & 1,0000 \\
\hline
\end{tabular}

Fonte: Dados registrados pela Cooperativa Beta

Efetuando uma análise prévia da Tabela 1 podemos observar que quase $30 \%$ das devoluções são em decorrência de avarias no transporte, 23,68\% são em função de emissão de notas fiscais incorre-

RGC, Santa Maria, v.2, n.4, Págs. 35-44, jul./dez. 2015 
tas e outros $34 \%$ por devolução de produto não justificado e devolução por desvio de qualidade. Ou seja, os dois principais motivos registrados podem ser solucionados se houver empenho das pessoas envolvidas. Já que, avarias no transporte podem ser corrigidas com baixo custo, através de um carregamento mais adequado ou com maior cuidado. Emissões de notas fiscais podem ser acertadas através de treinamento do emissor ou o acréscimo de uma inspeção final antes do despacho da mercadoria.

Os $17,5 \%$ de produtos devolvidos por desvio da qualidade somam o percentual que deve ser avaliado com mais critério, pois a perda da qualidade pode ocorrer por diversos motivos, como o próprio transporte incorreto ou erros na linha de produção. O segundo motivo é o que mais preocupa, já que, se há incoerências no processo produtivo esse percentual poderá ficar ainda mais significativo se medidas corretivas não forem tomadas com brevidade.
Os 19 produtos monitorados, foram subdivididos em 8 grupos diferentes para a análise das quantidades de material que retorna, do motivo das ocorrências, das ações tomadas pela beneficiadora, do setor responsável pelo ocorrido e das causas dessa devolução. Os grupos foram estabelecidos considerando o mesmo tipo de produto, ou seja, diferem apenas em quantidade de conteúdo e modelo de embalagem. Todavia, nos registros efetuados considerouse a quantidade de produto total, em quilos, o que facilitou a determinação dos grupos de produtos. As devoluções foram registradas de acordo com os pedidos que retornaram, por isso, em uma devolução podem-se observar vários produtos.

Os grupos foram identificados pelas letras maiúsculas de $\mathrm{A}$ a $\mathrm{H}$ de acordo com os registros fornecidos pela empresa. Em quilos de produto devolvido, em ordem decrescente de quantidade os dados apresentaram os resultados conforme a Tabela 2.

Tabela 2: Frequência relativa dos produtos devolvidos em relação ao total registrado.

\begin{tabular}{cccc}
\hline Produto & Soma & Frequência relativa & Frequência relativa $(\boldsymbol{\%})$ \\
\hline A & 1498853,78 & 0,6764 & 67,64 \\
C & 229954,00 & 0,1038 & 10,38 \\
B & 166586,60 & 0,0752 & 7,52 \\
D & 153132,00 & 0,0691 & 6,91 \\
E & 115239,88 & 0,0520 & 5,20 \\
G & 28849,00 & 0,0130 & 1,30 \\
H & 21266,00 & 0,0096 & 0,96 \\
F & 1924,00 & 0,0009 & 0,09 \\
\hline Total & 2215805,26 & 1,0000 & 100,00 \\
\hline
\end{tabular}

Fonte: Dados registrados pela Cooperativa A

Com a observação das frequências relativas de devolução dos produtos já é possível identificar que a ênfase do estudo deve ser dada ao grupo A. Pois nota-se que em $67,64 \%$ dos casos de devoluções são de produtos desse grupo, somando aproximados $1.500 .000,00 \mathrm{Kg}$ no período.

Tem-se no grupo A um produto com cinco embalagens diversas, aproximadamente $68 \%$ dos apontamentos de devolução correspondem a esses artigos, são no total quinhentos e setenta casos. Destes, duzentos e quarenta e nove representam avarias ou falta de produto no transporte e carregamento, ou seja, aproximadamente $44 \%$, cento e noventa e oito são devoluções dos clientes, dentre elas cento e quatorze por desvio de qualidade, em torno de $35 \%$ dos registros, estes dois casos representam 78,4\%. Os outros $21,6 \%$ correspondem a cento e uma notas fiscais incorretas, quatorze desistências de pedido, três sinistros e duas recusas por atraso na entrega. De acordo com estes números será necessária uma reavaliação das formas de transporte, pois neste grupo em específico são em torno de cinquenta e seis mil quilos de produto.

O grupo B é formado por um produto com três formas diferentes de envase. Somam cento e oito retornos de mercadoria, sessenta devoluções de produto pelos clientes, destas quarenta por desvio de qualidade e duas por prazo de validade ultrapassado, vinte e três avarias ou faltas no transporte, outras vinte e uma emissões de notas fiscais erradas, três casos de desistência do pedido e um sinistro. Em torno de $40 \%$ dos registros poderiam ser resolvidos pela indústria internamente. 
São cinco produtos que compõe o grupo $\mathrm{C}$, que diferem entre si pela quantidade que contém cada embalagem. Foram noventa e oito devoluções, destacam-se cinquenta notas fiscais incorretas, ou seja, mais da metade dos registros para o grupo, vinte e quatro devoluções de produto pelo cliente, das quais seis são por desvio da qualidade contratada e duas por prazo de validade expirado, onze avarias de transporte, dez envios de produto diferentes dos solicitados, uma avaria ou falta no carregamento, uma desistência pelo cliente e um sinistro. Com trabalhos preventivos, grande parte dos episódios poderia ser evitada.

Compreendem ao grupo D dois produtos similares que registram cinquenta e quatro casos, uma troca de produto, uma avaria por transporte falho, uma desistência de compra por parte do cliente, treze notas fiscais emitidas incorretamente e trinta e oito devoluções de produto pelo cliente sendo quatorze por desvio da qualidade. Neste caso poderiam ser reavaliados cinquenta por cento dos casos registrados.

Abrange o grupo $\mathrm{E}$ um só produto, mas dezoito devoluções, destas dez são devoluções de cliente e em duas delas por desvio de qualidade. Nos outros oito casos um refere-se a avaria no transporte e sete são notas fiscais com erro na emissão. Quer dizer, oito acontecimentos que somam cinquenta e oito mil quilos poderiam ser revistos internamente.

Corresponde ao grupo $\mathrm{F}$ um produto apenas que teve quatro retornos para a Cooperativa, três deles em função de devoluções dos clientes e uma emissão de nota fiscal incorreta. Percentualmente é o grupo que interfere menos no saldo total de quilos de produto devolvido, contudo pelo menos uma das restituições poderia ter sido reduzida, que é o caso da emissão da nota fiscal.

No grupo $G$ tem-se um produto e duas devoluções, uma delas por devolução do cliente e a outra emissão de nota fiscal errada. Apesar da pouca quantidade de devoluções, a quantidade de produto devolvido chega a quase vinte e nove mil quilos, ou seja, um prejuízo bem expressivo.

$\mathrm{O}$ grupo $\mathrm{H}$, é composto por um único produto. Do total de devoluções, para este produto foram registradas sete ocorrências, sendo quatro devoluções do cliente, uma delas por estar fora do prazo de validade, uma avaria no transporte e duas emissões de nota fiscal incorreta. Ou seja, de sete incoerências pelo menos três poderiam ser evitadas com medidas paliativas.

É importante considerar que parte dos produtos ou pedido que são devolvidos pelo cliente sem qualquer avaria e em perfeitas condições de consumo são novamente inseridos ao mercado para consumo. Essa reinserção ao mercado reduz o custo e as perdas da Cooperativa, todavia o gasto em logística é irreversível, bem como os prejuízos a sua imagem.

Através da análise descritiva dos grupos foi possível observar as seguintes medidas:

Tabela 3: análise descritiva dos grupos de produtos

\begin{tabular}{lcccccccc}
\hline \multicolumn{1}{c}{ Grupos } & $\mathbf{A}$ & $\mathbf{B}$ & $\mathbf{C}$ & $\mathbf{D}$ & $\mathbf{E}$ & $\mathbf{F}$ & $\mathbf{G}$ & $\mathbf{H}$ \\
\hline Soma & 1498854 & 166586,6 & 229954 & 153132 & 115239,9 & 1924 & 28849 & 21266 \\
Média & 2629,57 & 1542,47 & 2346,47 & 2835,78 & 6402,22 & 481 & 14424,5 & 3038 \\
Mediana & 125 & 20 & 150 & 299 & 28,5 & 311,5 & 14424,5 & 240 \\
Moda & 10 & 10 & 40 & 12960 & 1 & 1 & & \\
Variância & 41907305 & 36331328 & 30277718 & 21972956 & $1,38 \mathrm{E}+08$ & 383814 & 37247081 & 29712336 \\
Desvio padrão & 6473,59 & 6027,55 & 5502,52 & 4687,53 & 11748,53 & 619,53 & 6103,04 & 5450,90 \\
Erro padrão & 271,15 & 580,00 & 555,84 & 637,89 & 2769,15 & 309,76 & 4315,5 & 2060,25 \\
Assimetria & 3,09 & 4,74 & 3,21 & 1,52 & 1,47 & 0,93 & & 2,32 \\
Curtose & 8,99 & 22,98 & 10,06 & 0,66 & 0,19 & $-0,91$ & 129 & 5,59 \\
Intervalo & 34999,4 & 39999,6 & 26999,6 & 12957 & 29259 & 1299 & 8631 & 14999 \\
Mínimo & 0,6 & 0,4 & 0,4 & 3 & 1 & 1 & 10109 & 1 \\
Máximo & 35000 & 40000 & 27000 & 12960 & 29260 & 1300 & 18740 & 15000 \\
N & 570 & 108 & 98 & 54 & 18 & 4 & 2 & 7 \\
\hline
\end{tabular}

Fonte: Planilha eletrônica

A primeira linha da Tabela 3 indica o somatório de produtos que retornaram para a indústria em qui- los. Efetuando o somatório geral obtêm-se mais de dois milhões de quilos de produto envolvido em 
processo de retorno para a indústria em um período de dezenove meses, ou seja, são mais de cem mil quilos ao mês em uma média geral. Como analisado anteriormente há a possibilidade de reduzir esse número acrescentando maiores cuidados na emissão de notas fiscais e no transporte e armazenamento dos produtos acabados.

Pela análise das medidas de tendência central percebe-se que a variação dos dados é grande em todos os grupos. Em relação a média dos grupos, os desvios padrões possuem valores bem altos o que indica a grande dispersão dos dados. As médias também apresentam alta variabilidade conforme os resultados do erro padrão.

Através da amplitude apresentada tem-se como retorno que a quantidade mínima e a máxima de produtos em quilos, ou seja, o intervalo é a diferença e os valores de mínimo e máximo significam o intervalo a ser representado graficamente. Também se pode observar que os pedidos que apresentaram devolutas são tanto aqueles com grandes quantias quanto os com poucos quilos de produtos.

$\mathrm{O} \mathrm{N}$ representa o número total de observações feitas por produto, o total de registros do período é de 760 devoluções, porém como em alguns casos há mais de um produto por pedido o somatório da Tabela 3 é de 871 ocorrências.

\section{Conclusão}

Os problemas com logística são muito comuns nas empresas e causam prejuízos bem significativos se não forem evitados ou trabalhados. No caso da cooperativa estudada foi possível observar que os problemas com questões que envolvem os processos operacionais logísticos da indústria de beneficiamento dos produtos tende a ser uma questão que favorece a redução do lucro final.

A razão citada com maior frequência como sendo o motivo da devolução foi a avaria ou falta em função do transporte, em 29,74\% dos 760 registros. Ou seja, é importante que a cooperativa reveja o processo de entrega e acondicionamento dos produtos. Sugere-se que todo o processo seja verificado para que se encontre o ponto crítico. Podem ser inclusos no mesmo processo os outros $6,45 \%$ mencionados como avaria ou falta no carregamento. Efetuando a soma de forma indiscriminada em quilos de produto se obtêm $51.457,8 \mathrm{Kg}$ que deixam de se tornar lucro, porém como foi produzido torna-se um custo.

Outro indicador que apresentou bastante relevância na análise foi de emissão de nota fiscal incorreta, que totalizou $23,68 \%$ dos apontamentos. Assim como o item anterior a sugestão é a reavaliação do processo que envolve as emissões de notas. Podem ser criados treinamentos de pessoal, conscientização dos envolvidos, principalmente porque a emissão de notas fiscais envolve ainda questões relacionadas a pagamentos de impostos que podem gerar problemas maiores ao setor contábil da Cooperativa. Além disso, a devolução significa custo e redução direta da lucratividade, já que, há a tentativa de atendimento do pedido, o retorno dessa mercadoria para a indústria e a nova entrega do pedido de forma correta. Ou seja, esse erro causa retrabalho e no mínimo um deslocamento desnecessário, bem como todos os custos envolvidos nesse processo.

A devolução do produto por parte dos clientes somou no total $34,08 \%$, sendo que $17,5 \%$ foram especificadas as devolutas por desvio da qualidade. Propõe-se a análise detalhada dos casos relatados de desvio de qualidade, a verificação do motivo pelo qual o produto não apresenta a qualidade habitual pode demonstrar tanto problemas complexos, como desordem na linha de fabricação quanto a necessidade de pequenos ajustes de transporte e armazenamento.

Os demais motivos assinalados somam 6,05\%, devem ser considerados tão importantes quanto os demais, contudo sua repetição no período é inferior. Entre esse percentual $2,37 \%$ é referente a desistência da compra, seria interessante a averiguação da razão para o cliente deixar de adquirir o produto. Poderiam ser oferecidas condições favoráveis, promoções ou alguma vantagem que fizessem com que este cliente tornasse a fazer parte da carta de clientes nos casos de rompimento definitivo.

Com auxílio da estatística descritiva foi possível interpretar os dados referentes a devoluções de produtos e perceber com maior clareza os pontos mais frequentes. A reavaliação de alguns processos e a reestruturação de pontos mais críticos pode auxiliar muito a indústria na redução de perdas com processos operacionais logísticos. Desta maneira, todo o custo gerado por retrabalhos pode ser reinvestido na própria Cooperativa para melhorias internas e assim agregar maior valor ao cooperados. 


\section{Referências}

ANTONIALLI, L. M. Influência da mudança de gestão nas estratégias de uma cooperativa agropecuária. Rev. adm. contemp. vol.4 n.1 Curitiba Jan./Apr. 2000.

BARBETA, P. A.; REIS, M. M.; BORNIA, A. C. Estatística para cursos de engenharia e informática. São Paulo: Atlas, 2004.

BERETTA, S. Unleashing the integrationn potential of ERP system. Business Process Management Journal, v. 8, n. 3, p. 254-277, 2002.

BIJMAN, J.; ILIOPOULOS, C. Farmers' cooperatives in the EU: policies, strategies and organization. Annals of Public and Cooperative Economics 85: 4 2014, pp. 497-508.

BRUSSELAERS, J.; POPPE, K.; AZCARATE, T. G. Do policy measures impact the position and performance of farmers' cooperatives in the EU? Annals of Public and Cooperative Economics 85:4 pp. 531-553, 2014.

DAVENPORT, T. H. Process inovation. Boston: Harvard Business School Press, 1993.

GIL, A. C. Gestão de pessoas. São Paulo: Atlas, 2001.

GONÇALVES, J. E. L. Processo, que processo? Revista de administração de empresas, v. 40, n. 4, p. 8-19, Out./Dez. 2000.

JESTON, J.; NELIS, J. Business process management: practical guidelines to successful implementations. Oxford: Elsevier, 2006.

IRITANI, D. R. et al. Análise sobre os conceitos e práticas de gestão por processo: revisão sistemática e bibliometria. Gest. Prod. vol.22 no.1 São Carlos Jan./Mar. 2015.

LARSON, R; FARBER, B. Estatística Aplicada. 4 ed. São Paulo: Pearson Prentice Hall, 2010.
MALHOTRA, N. K. Pesquisa de marketing: uma orientação aplicada. 4ed. Porto Alegre: Bookman, 2006.

MORI, P. A. Community and cooperation: the evolution of cooperatives towards new models of citizens' democratic participation in public services provision. Annals of Public and Cooperative Economics. 85:3, pp. 327-352, 2014.

MÜCKENBERGER, E. et al. Gestão de processos aplicada à realização de convênios internacionais bilaterais em uma instituição de ensino superior pública brasileira. Produção, v. 23, n. 3, p. 637651, jul./set. 2013.

OLIVEIRA, S. B. Qualificando os processos de sua organização. In: VALLE, R.; OLIVEIRA, S. B. (Org.). Análise e modelagem de processos de negócio: foco na notação BPMN (Business Process Modeling Notation). São Paulo: Atlas, 2009.

PÁDUA, S. I. D. Estudo sobre a aplicação do método de avaliação do modelo de processo de negócio do EKD. Produção, v. 22, n. 1, p. 155-172, jan./fev. 2012.

RICHARDSON, R. J. Pesquisa social: métodos e técnicas. 3. ed. São Paulo: Atlas S. A., 1999.

SILVA, E. M. da; GONÇALVES, V.; MUROLO, A. C. Estatística para cursos de: economia, administração e ciências contábeis. 3. ed. São Paulo: Atlas S. A., 1999. 
